\title{
Tendencias en metodología de investigación en Psicoterapia: Una aproximación epistemométrica*
}

\section{Trends in research methodology in Psychoterapy: An epistemometric approach}

\author{
Alejandro Cifuentes-Muñoz \\ ORCID 0000-0003-1767-5517 \\ Universidad de Talca, Chile \\ Recibido: 12 de octubre de 2018 \\ Revisado: 6 de noviembre de 2018 \\ Aceptado: 20 de enero de 2019
}

\section{Resumen}

El objetivo del presente artículo es describir las principales tendencias metodológicas en investigación psicoterapéutica de los últimos cinco años, como una forma de responder a "cómo se está conociendo lo que se conoce" en la disciplina. La descripción giró en torno a tres variables: tipos de problemas de investigación, tipos de investigación y tipos de enfoque de investigación. La metodología utilizada se centró en un análisis descriptivo epistemométrico respecto a la base de datos Web of Science. La muestra fue no probabilística y correspondió a 46 artículos de investigación en psicoterapia publicados durante el periodo 2013 y 2017. Se encontró que la mayoría de los artículos abordan problemas prácticos (52.2\%), fueron investigaciones de tipo descriptivo (54.3\%) y utilizaron un enfoque cualitativo (45.7\%). Finalmente, se concluye que los investigadores en psicoterapia realizan, principalmente, preguntas de tipo práctico en torno al tratamiento de pacientes, obteniendo respuestas de alcance limitado, centradas en fenómenos particulares. Se discuten las limitaciones del estudio sobre la generalización de los resultados.

Palabras clave: Metodología de investigación, Psicoterapia, Epistemometría, Epistemología.

Artículo de Investigación. Cómo citar: Cifuentes-Muñoz, A. (2019). Tendencias en metodología de investigación en Psicoterapia: Una aproximación epistemométrica. Diversitas: Perspectivas en Psicología, 15(2), 201-210. DOI: 10.15332/22563067.3856

Correspondencia: Alejandro Cifuentes-Muñoz, Universidad de Talca, Chile. Dirección postal: Avenida tircay s/n Universidad de Talca. Correo electrónico: acifuentes@utalca.cl 


\section{Abstract}

The aim of this article is to describe the main methodological trends in psychotherapy research in the past five years, as a way of responding to "how it is knowing what is known" in the discipline. The description focused on three variables: types of research problems, types of research and types of research approach. The methodology used in this article focuses on a descriptive epistemometric analysis regarding the Web of Science database. The sample was non-probabilistic and corresponded to 46 psychotherapy research articles published between 2013 and 2017. It was found that most articles dealt with practical problems (52.2\%), descriptive research $(54.3 \%)$ and approach reality through a qualitative approach $(45.7 \%)$. Finally, it is concluded that researchers in psychotherapy mainly ask practical questions about the treatment of patients, obtaining answers of limited scope, focusing on phenomena. The limitations of the study on the generalization of results are discussed.

Keywords: Research Methodology, Psychotherapy, Epistemometric, Epistemology.

\section{Introducción}

¿Cómo conocemos lo que conocemos en una disciplina? Si bien es una reiterada pregunta en torno a la filosofía de la ciencia o epistemología, la respuesta no es clara, y por cierto, tampoco exenta de controversias, particularmente en lo que compete a la Psicoterapia. Es una pregunta que suele ser eludida en Psicoterapia, así como en otras disciplinas, debido a que se considera que tales reflexiones no son la vía más rápida, o la que más aporte, al estatus científico de la misma. Estatus que por cierto aún no se alcanza (Opazo, 1992). Otra forma de entender la pregunta planteada es refiriéndose a aquello que nos permite conocer un determinado fenómeno en una disciplina, es decir, la metodología.

En consecuencia, es posible llevar a cabo una breve revisión histórica sobre los aspectos metodológicos de la investigación en Psicoterapia. Sales (2009) señala que el desarrollo en investigación psicoterapéutica comenzó con los métodos naturalistas, es decir, el análisis cualitativo del caso a caso en contexto real. Luego, tomó predominancia, incluso hasta la actualidad, la investigación de resultado (outcome reasearch), centrada en los efectos de la psicoterapia y no en su proceso (Hill \& Corbett, 1993). De esta manera, se enfatiza en la cuantificación de los resultados junto con el control experimental, adoptándose el paradigma positivista, predominante en la ciencia moderna, lo que da lugar a los estudios analógicos (analogue reasearch). Es decir, la investigación de los tratamientos psico- terapéuticos mediante la simulación en laboratorio. Al final de la década de los 70, los ensayos clínicos, o estudios de resultado llevados a contextos terapéuticos reales, cobraron relevancia (Sales, 2009). Finalmente, y con base en las necesidades del contexto político-económico de los Estados Unidos, en los 90 , se vuelve necesario establecer una clasificación de los tratamientos con soporte empírico (Empirically Supported Treatments). Esta tarea fue realizada por la American Psychological Association (APA) sentando el norte de la investigación en psicoterapia y de lo que sería considerado ciencia en tal disciplina, así como las aspiraciones metodológicas que rigen hasta la actualidad (Rodríguez, 2004).

Sin embargo, hacer un breve recorrido histórico sobre la metodología de investigación en psicoterapia, si bien otorga un panorama interesante, no parece ser suficiente para aproximarse a una respuesta a la pregunta introductoria, y si además se considera el contexto geográfico y cultural, la situación es más compleja. Latinoamérica es una región que no se caracteriza por entregarle valor a la ciencia (Moyano, 1999), por ende, la producción científica que aporte luces como respuesta a la pregunta planteada en un inicio, es mínima. En consecuencia, los investigadores en psicoterapia normalmente no se interesan por el marco epistemológico de la metodología de investigación en la que se fundamentan sus hallazgos. Les preocupa "conocer" pragmáticamente un fenómeno, por ejemplo, un determinado tratamiento frente a una determinada psicopatología, no el "cómo se 
conoce" lo que se conoce de aquel fenómeno, de tal manera, y a pesar de servirse evidentemente de ella para cada investigación, no hay un foco puesto en el marco epistemológico de la metodología.

Metodología, "designa el modo o estilo de afrontamiento que tenemos respecto a la realidad, respecto de cómo formulamos los problemas y buscamos respuestas" (Moyano, 1999, p. 2). Es decir, a priori se aprecian tres elementos clave en tal definición: una preocupación por las preguntas, por las respuestas y por el enfoque de acercamiento a la realidad. En estos tres elementos referidos se basa el desarrollo del presente artículo, como forma de aproximarse a la respuesta de la pregunta planteada al inicio. No son pocas las clasificaciones en metodología de investigación, existiendo numerosos manuales, libros y publicaciones, donde cada autor pone al acento en algún criterio que permita agrupar a ciertos miembros en una categoría, de forma tal de facilitar su comprensión (para estudios con estas características véase Bunge \& Ardila, 1988; Hernández, Fernández, \& Baptista, 2014; Sierra Bravo, 1987). No es objeto de este artículo presentar tales clasificaciones, sin embargo, se considera que a lo menos tres focos teóricos abordarían y responderían, al menos parcialmente, a los tres aspectos de la metodología definidos, y que son susceptibles de aplicar a diversas disciplinas, particularmente a la que compete en este estudio. Éstos son: los tipos de problemas de investigación, los tipos de investigación y finalmente, los tipos de enfoques de investigación, cada uno respondiendo a otras tres preguntas fundamentales.

1. ¿Qué preguntas se formulan en psicoterapia? Se cree que la respuesta está dada por los tipos de problemas de investigación en los que se pone el acento, es decir, qué aspectos de la realidad emergen como cuestionamientos en los investigadores en psicoterapia. Bunge y Ardila (1988) plantean tres preguntas o problemas típicos en psicología: empíricos, teóricos y prácticos. Los problemas empíricos responden principalmente a la medición, no obstante, también a la descripción de un fenómeno empírico, es decir ¿cuál es el valor o cualidad de X?. Los problemas teóricos, no tienen sustento empírico y se centran en la explicación, es decir ¿por qué
$X$ tiene la cualidad o valor $Y$ ?. Finalmente, los problemas prácticos se centran, por ejemplo, en el tratamiento de pacientes, es decir, ¿cómo se puede modificar el valor o cualidad de $\mathrm{X}$ ?

2. ¿Cuál es el alcance de las respuestas que se espera encontrar? Los tipos de investigación planteados por Hernández, Fernández y Baptista (2014), se considera, responderían a esta pregunta en la medida que esta clasificación, según los autores, se presenta como un continuo que abarca desde el menor al mayor alcance que pudiera tener una investigación. En esta clasificación se encuentran las investigaciones de tipo exploratorias, descriptivas, correlacionales y empíricas. Las investigaciones exploratorias se utilizan para abordar temas poco estudiados o innovadores, es decir, de objetos sobre los que se tiene poca información, por ende, no hay medición, y preparan el terreno para nuevos estudios con hipótesis y preguntas más refinadas. Las investigaciones descriptivas, como su calificativo lo expone, se centran en la descripción de variables, es decir, especifican las propiedades y características importantes de un fenómeno. Las investigaciones correlacionales pretenden conocer, mediante la cuantificación, la relación o grado de asociación entre dos o más conceptos, categorías o variables, siendo su poder explicativo, bastante limitado. Finalmente, las investigaciones explicativas, determinan las causas de los fenómenos, buscando explicar por qué ocurre un fenómeno o por qué se relacionan dos o más variables, siendo característica su alta estructuración y su alta relación con la experimentación.

3. ¿Cómo nos aproximamos a la realidad? Sin duda, y evidentemente no de forma exclusiva, esta respuesta está mediada por los enfoques de investigación. En la actualidad es posible dar cuenta de al menos tres: cuantitativo, cualitativo y mixto. Sierra Bravo (1987) en torno a la investigación social, refiere que el carácter cuantitativo, se centra en los aspectos objetivos y susceptibles de ser cuantificados, siendo predominantemente inductivo y nomotético. Por su parte el carácter cualitativo, busca descubrir y comprender el sentido y significado de los fenómenos, centrándose en lo ideográfico 
y particular. Por su parte, las aproximaciones mixtas (Jonhson \& Onwuegbuzie, 2004), son una mezcla de ambos enfoques a través de las etapas de investigación (modelo mixto) o bien, mediante la inclusión de una fase cualitativa y una cuantitativa en un estudio (método mixto).

En la figura 1 se puede apreciar, a modo de síntesis y orientación posterior, los fundamentos teóricos del presente artículo.

¿Cómo conocemos lo que conocemos?

\section{Metodología}

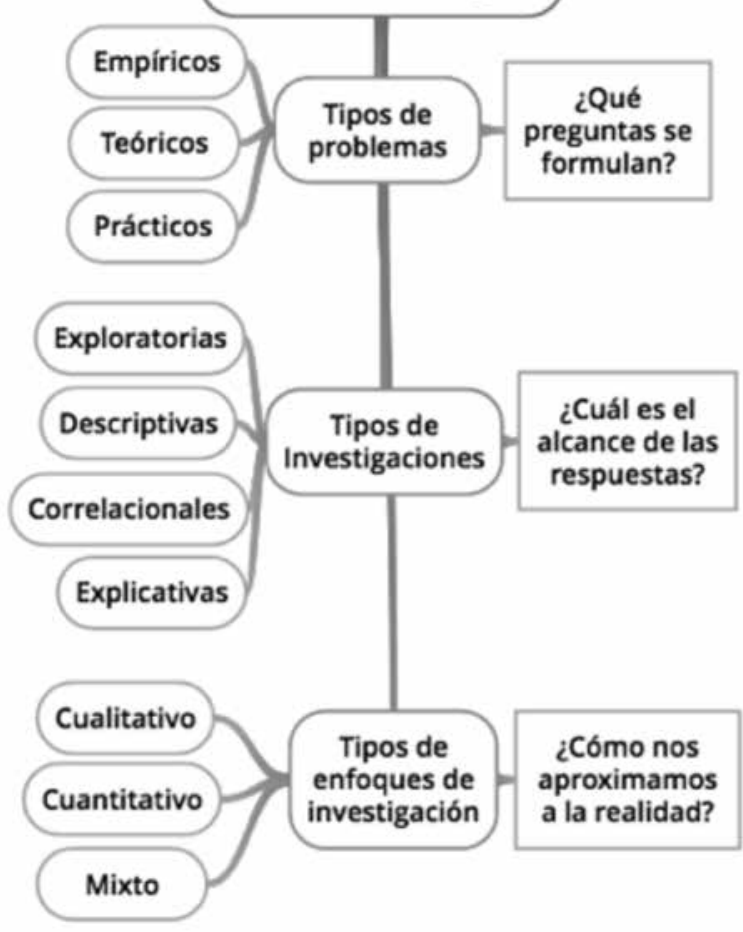

Figura 1. Cuadro de resumen

Fuente: elaboración propia.

Con el desarrollo tecnológico en el campo del conocimiento científico en la actualidad es posible responder a las preguntas planteadas, o presentar al menos una aproximación, a través de un análisis cuantitativo de la información o producción científica. Este enfoque o forma de tratar los datos se denomina cientometría, o con su correcto marco etimológico, epistemometría. Krauskopf (1994) establece que ésta se ocupa del "análisis de los aspectos cuantitativos de la generación, propagación y utilización de la información científica, a fin de contribuir a una mejor comprensión del mecanismo de la investigación científica como actividad social" (p. 426). Esta aproximación ha sido ampliamente utilizada en las tres últimas décadas, para el análisis de la producción científica, por ejemplo, de las revistas y artículos indexados en las bases de datos nacionales e internacionales (para estudios con estas características véase Agudelo, Bretón \& BuelaCasal, 2003; Alcain \& Lascurain, 2002; Krauskopf \& Pratt, 1990; Krauskopf \& Krauskopf, 2008; Lillo \& Martini, 2013; Moyano \& Moyano, 1996; Moyano \& Ramos, 2000; Polanco-Carrasco, Gallegos, Salas \& López-López, 2017; Rey, Martínez \& Guerrero, 2009; Riffo, 2011; Rull \& Pereda, 2011; Salas et al., 2017; Vera-Villarroel \& Lillo,2006; Vera-Villarroel \& Mustaca,2006).

En consecuencia, el presente artículo pretende dar una aproximación a las respuestas de las tres preguntas realizadas, que emergen de la definición de metodología, y contribuir a describir la forma en que se está conociendo lo que se conoce en psicoterapia. Por consiguiente, el objetivo de este artículo es describir cuáles son las principales tendencias metodológicas en la investigación psicoterapéutica basándose en los tipos de problemas científicos, los tipos de investigación y los enfoques de investigación que se aprecian en las producciones científicas de los últimos 5 años, llevándose a cabo mediante una aproximación epistemométrica sobre la base de datos Web of Science.

\section{Método}

\section{Unidad de análisis}

Corresponde a los artículos publicados entre 2013 y 2017 cuyo resumen o texto completo es posible recuperar mediante la base de datos Web of Science en torno a criterios de búsqueda específicos en el campo de título y resumen (tema). La muestra inicial estaba conformada por 67 artículos de los cuales sólo 46 describían su metodología explícitamente y se configuraban como una investigación en psicoterapia. La muestra final quedó conformada por estos 46 artículos. Se acotó el rango a los últimos 
5 años de publicaciones en función de describir las tendencias metodológicas más actuales.

\section{Materiales}

La base de datos electrónica Web of Science perteneciente a Thompson Reuters, contiene información sobre investigación multidisciplinaria de alta calidad publicada en revistas líderes mundiales en las Ciencias, Ciencias Sociales, Artes y Humanidades. Para el presente artículo se utilizó la colección principal de Web of Science compuesta por Science Citation Index Expanded (SCI-EXPANDED), Social Sciences Citation Index ( $\mathrm{SSCl}$ ), Arts \& Humanities Citation Index (A\&HCl) y Emerging Sources Citation Index (ESCl), por ser considerados los índices más congruentes con la disciplina psicoterapéutica.

Se seleccionó la base de datos Web of Science ya que en la actualidad, los artículos publicados en tal base, son el indicador de mayor uso a nivel internacional para la medición de la productividad científica (Vera-Villarroel \& Lillo, 2006).

\section{Procedimiento}

La metodología seguida en este artículo es de tipo descriptiva epistemométrica mediante análisis de documentos. Se realizó un muestreo por conveniencia no probabilístico.

Se llevó a cabo un proceso de búsqueda avanzada para los productos científicos de la colección principal de la plataforma Web of Science, utilizando como criterio de búsqueda, todas las publicaciones que tuvieran el término "Psychotherapy" en el título y las que incluyeran la palabra "methodology" en el tema (abstract). Se filtraron únicamente las publicaciones que se incluyeran en la categoría "artículo” y se limitó la búsqueda al periodo 2013 al 2017. La fórmula de búsqueda avanzada fue la siguiente: (( $\mathrm{Tl}=$ Psychotherapy) AND (TS=methodology) AND (PY=2013-2017)) AND Tipos de documento: (Article). Índices=SCI-EXPANDED, SSCI, A\&HCI, ESCI.

Del universo total de publicaciones científicas en Web of Science, y mediante los criterios de búsqueda referidos, se acotó una muestra de 46 ar- tículos, todos en idioma inglés. Si bien existía una heterogeneidad importante en las revistas que albergan tales artículos, es importante referir que estas, en su mayoría, incorporan artículos cuantitativos, cualitativos y mixtos según lo establecido en sus normas de publicación. De estos 46 artículos cerca del $44 \%$ correspondió al área de Psicología Clínica, un 22\% al área de Psiquiatría, un 9\% al área de Psicología Multidisciplinaria, un 6\% al área de Psicología Aplicada y el $19 \%$ restante, a otras áreas 1.

Luego de una primera lectura, se utilizó el software SPSS (versión 23) con objeto de clasificar los artículos mediante 3 variables categóricas nominales: 1) tipos de problemas (teóricos, prácticos, empíricos), 2) tipos de investigación (exploratoria, descriptiva, correlacional y explicativa) y 3) tipo de enfoque de investigación (cuantitativo, cualitativo y mixto). Dentro del enfoque de investigación mixto se incluyó, sin discriminación, tanto los modelos como métodos mixtos. El criterio de clasificación se operacionalizó mediante las definiciones y categorías establecidas por Bunge y Ardila (1988) para tipos de problemas; por Hernández et al. (2014) para los tipos de investigación y por Sierra Bravo (1987) para los enfoques de investigación. Finalmente, se asignaron valores a cada variable categórica y se realizaron estadísticos descriptivos con el fin de obtener tablas cruzadas y gráficos de frecuencia.

\section{Resultados}

\section{Tipos de problemas de investigación}

Los estadísticos descriptivos de la Figura 2 muestran que un $52.2 \%$ de la muestra de artículos revisados abordan problemas de tipo práctico, seguido de los problemas teóricos con un $37 \%$ y los problemas empíricos con un $10.9 \%$.

\section{Tipos de investigación}

Respecto a los tipos de investigación, es posible dar cuenta que más de la mitad de los artículos de la muestra presentan una investigación de carácter descriptivo, correspondiendo a un $54.3 \%$ del total.

1 Datos calculados mediante la herramienta de análisis de resultados de la Web of Science 


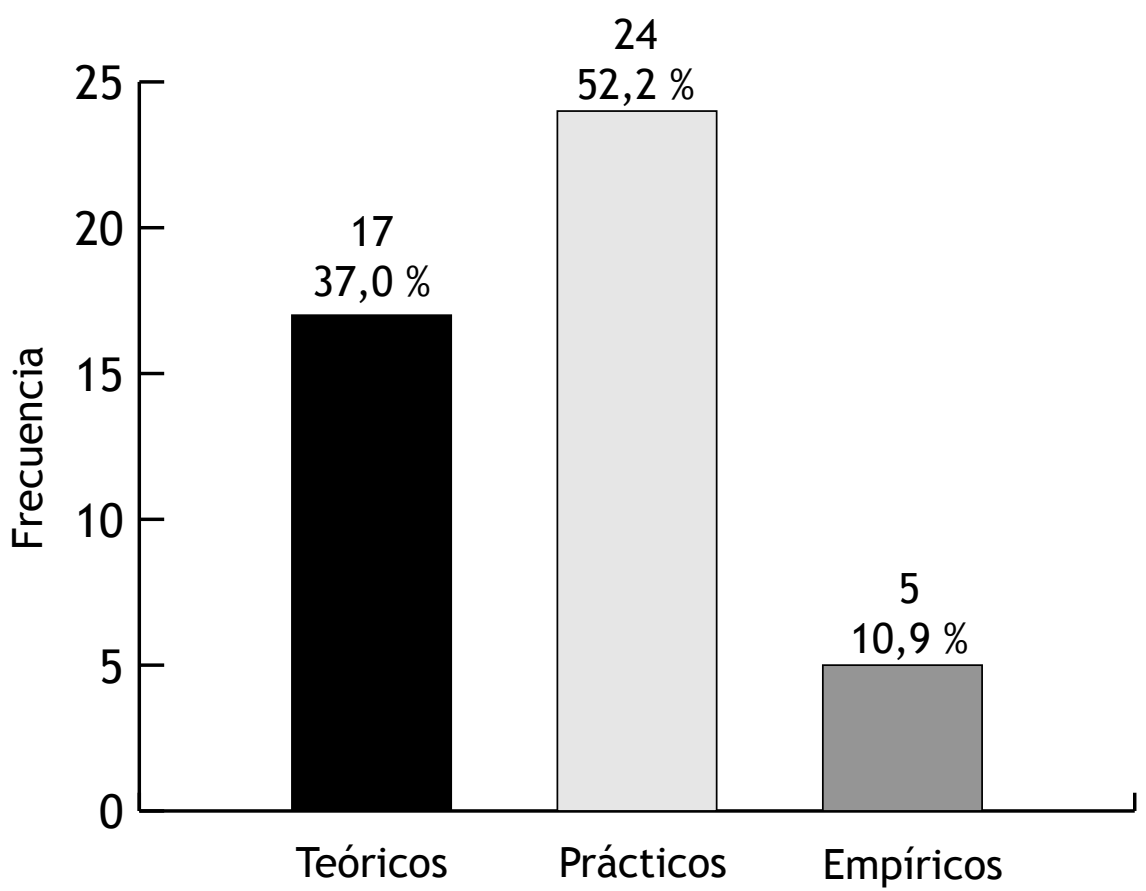

Figura 2. Tipos de problemas de investigación

Fuente: elaboración propia

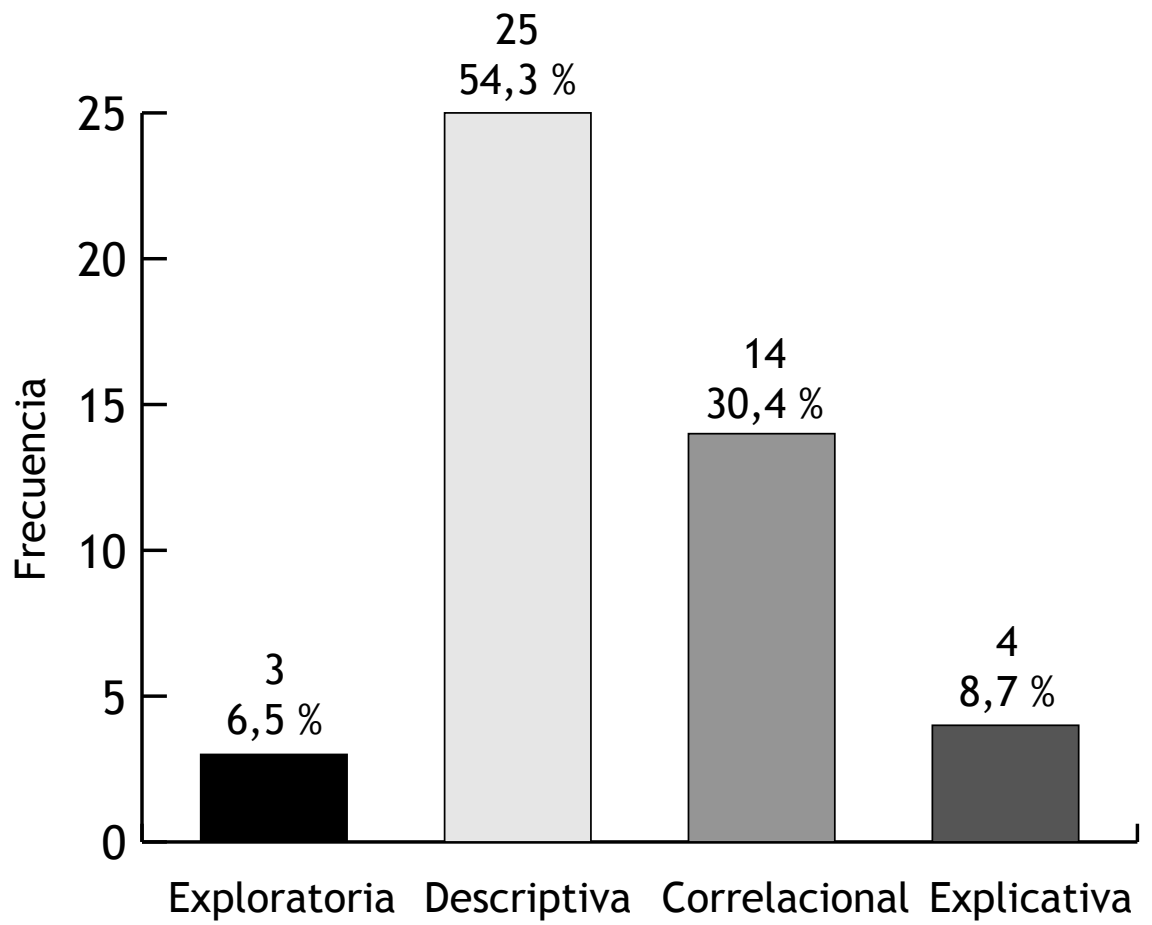

Figura 3. Tipos de investigación.

Fuente: elaboración propia. 
Le siguen las investigaciones de tipo correlacional con un $30.4 \%$, para finalizar con las de tipo explicativa y exploratoria con un $8.7 \%$ y $6.5 \%$ respectivamente. Véase la figura 3.

\section{Tipo de enfoque de investigación}

En torno al tipo de enfoque de investigación que se configura como predominante en la muestra seleccionada, y tal como se aprecia en la Figura 4, se puede referir que el $45.7 \%$ corresponde a enfoques de investigación cualitativos. Un $37 \%$ de los artículos presenta un enfoque cuantitativo y un $17.4 \%$ un enfoque mixto.

Respecto a la distribución de la variable tipos de problema de investigación por tipo de investigación, es posible decir que un $76.5 \%$ ( 13 artículos) del total de artículos teóricos, corresponde a un tipo de investigación descriptiva y que el $83.4 \%$ (20 artículos) de los artículos que abordan problemas prácticos corresponden a tipos de problemas descriptivos y correlacionales con un $41.7 \%$ cada uno.
En lo que refiere a la distribución de la variable tipos de problema por tipos de enfoque de investigación, se puede referir que el $70.6 \%$ de los artículos teóricos presentan un enfoque de investigación cualitativo. Respecto a los problemas de tipo práctico se presenta una distribución más homogénea en torno a los enfoques cualitativos, cuantitativos y mixtos con un $37.5 \%, 33.3 \%$ y $29.2 \%$ respectivamente. El $80 \%$ (4 artículos) de los artículos que abordan problemas empíricos presentan un enfoque cuantitativo.

Finalmente, respecto a la distribución del tipo de enfoque de investigación por el tipo de investigación es posible decir que el $85.7 \%$ (18 artículos) de los artículos con un enfoque cualitativo son de tipo descriptivo, así como el 52.9\% (9 artículos) de los artículos con enfoque cuantitativo son de tipo correlacional, seguido por un $47 \%$ que se distribuye de forma igualitaria en las investigaciones de tipo descriptivo y correlacional. Finalmente, los enfoques mixtos se distribuyen con un $62.5 \%$ y un $37.5 \%$ en investigaciones de tipo correlacionales y descriptivas, respectivamente.

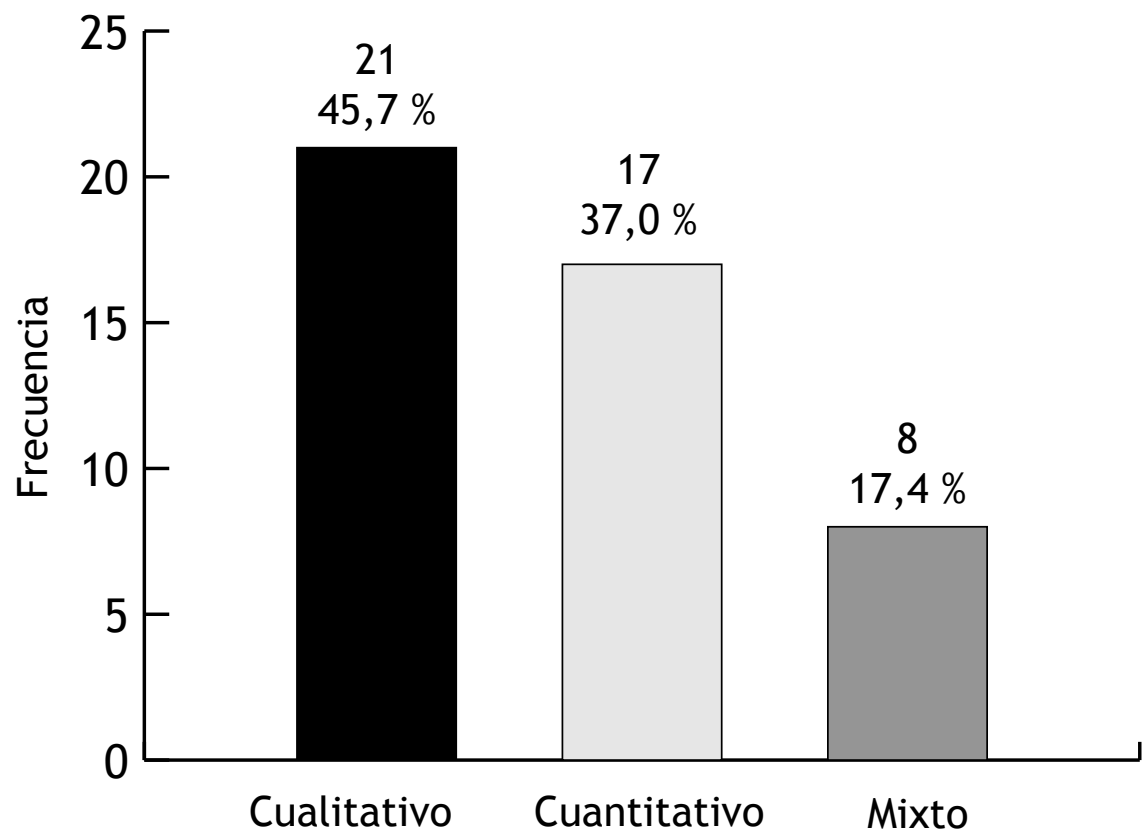

Figura 4. Tipo de enfoque de investigación

Fuente: elaboración propia. 


\section{Discusión}

El estudio presentado fue de tipo descriptivo y con un muestreo por conveniencia no probabilístico, por ende, previo a establecer cualquier conclusión respecto a los datos, es necesario tener en cuenta las limitaciones propias de estos estudios, tomando con cautela las interpretaciones de los datos y la representatividad de la muestra respecto a la población de artículos.

No obstante, y teniendo claro lo anterior, del estudio se pueden relevar interesantes reflexiones y respuestas tentativas a las preguntas planteadas con anterioridad.

¿Qué preguntas se formulan en psicoterapia? Según se aprecia en los datos obtenidos de esta muestra, los investigadores en psicoterapia, en su mayoría, están centrados en abordar problemas de tipo práctico, por ende, son precisamente ese tipo de preguntas las que se formulan, las cuales están principalmente centradas en la modificación de la cualidad o valor de un fenómeno. Es decir, y tal como establece Bunge y Ardila (1988), en torno al tratamiento de pacientes. Esto es esperable, ya que la psicoterapia, como disciplina de la psicología encargada del abordaje del sufrimiento humano, es eminentemente práctica, razón por la cual busca generar cambios precisamente en tal sufrimiento o psicopatología.

¿Cuál es el alcance de las respuestas que se espera encontrar? Los artículos de la muestra señalan que las respuestas a las preguntas que se formulan los investigadores en psicoterapia, están centradas en la descripción de las variables, de sus propiedades y características, por lo que en consecuencia con Hernández et al. (2014), el alcance que se espera es limitado. La figura 3 muestra que las investigaciones de tipo exploratorias $(6.5 \%$ ) y las explicativas (8.7\%), por lo cual, manteniendo la mesura de no rebasar los datos de la muestra, podría decirse que no hay una inclinación en los últimos 5 años hacia estos artículos. Esto implica que no hay una tendencia a investigaciones innovadoras -tipo exploratorias-, como tampoco hay una tendencia de buscar las causas de los fenómenos o variables en psicote- rapia mediante investigaciones estructuradas $-\mathrm{ti}$ po explicativas-. Lo anterior, podría relacionarse con el estancamiento del estatus científico de la psicoterapia, ya que los esfuerzos están centrados en artículos de descripción, como este estudio por ejemplo, y en artículos de correlación de variables con alcance explicativo limitado, carentes de experimentación rigurosa.

¿Cómo nos aproximamos a la realidad? Hay una tendencia de los artículos revisados, de aproximarse a la realidad centrándose en lo idiosincrático y particular, sin la búsqueda de leyes generales sobre el objeto de estudio psicoterapéutico, sino acentuando en la comprensión del fenómeno. Esta tendencia de la muestra se contrapone con lo establecido por Rodríguez (2004) respecto al énfasis en la experimentación y cuantificación, dada por la necesidad de dar un soporte empírico a los tratamientos en psicoterapia. Por ende, es interesante que el enfoque cualitativo de aproximación a la realidad prime sobre lo cuantitativo. Sin embargo, se considera que la aproximación por la que se opta en un artículo responde directamente al problema o pregunta de investigación que se quiere resolver. De tal manera, el $70.6 \%$ de los artículos que abordan problemas prácticos, correspondientes a más de la mitad del total de artículos de la muestra, presentan una aproximación cualitativa. Probablemente, de haber presentado la muestra seleccionada mayores tasas de problemas empíricos, la metodología hubiera sido otra. Sin embargo, no es posible ceñirse a tal aseveración como una certeza debido a las limitaciones del estudio.

Sin duda, la extrapolación de los resultados de este estudio es limitada, por lo que es necesario ampliar los criterios de búsqueda, las bases de datos y el tamaño muestral para tener conclusiones que tengan un mayor impacto y representación en la población total de artículos científicos. No obstante, el ejercicio presentado en este estudio otorga algunas bases acotadas y aproximadas, respecto a cómo responder algunas preguntas, habitualmente incómodas y que normalmente se eluden, en relación a la manera en que se conoce lo que se conoce en psicoterapia, es decir, al marco epistemológico de la metodología de investigación en tal disciplina. 


\section{Referencias}

Agudelo, D., Bretón-López, J., \& Buela-Casal, G. (2004). Análisis bibliométrico de las revistas relacionadas con Psicología de la Salud editadas en castellano. Salud Mental, 27(2), 70-85.

Alcain, M. \& Lascurain, M. (2002). Análisis de las revistas latinoamericanas de Psicología incluidas en el directorio Latindex. Papeles Del Psicólogo, 83, 34-43.

Bunge, M. \& Ardila, R. (1988). Filosofía de la psicología. Ed. Ariel, S. A. Barcelona.

Hernández, R., Fernández, C., \& Baptista, P. (2014). Metodología de la investigación. (6ta edición) Ed. McGraw Hill. México

Hill, C. \& Corbett, M. (1993). A perspective on the history of process and outcome research in counseling psychology. Journal of Counseling Psychology 40(1), 3-24.

Jonhson, R. \& Onwuegbuzie, A. (2004). Mixed methods research: A research paradigm whose time has come. Educational Researcher, 33(7), 14-26. DOI: 10.3102/0013189X033007014

Krauskopf, M. (1994). Epistemometria, a term contributing to Express the meaning and potential methodologies of Scientometrics in Spanish-speaking countries. Scientometrics, 30(2-3), 425-428.

Krauskopf, M. \& Krauskopf, E. (2008). Una mirada epistemométrica de la Revista Médica de Chile y su aporte al conocimiento en Medicina. Revista Medica de Chile, 136(8), 1065-1072. DOI: $10.4067 /$ S0034-98872008000800016

Krauskopf, M. \& Prat, A. (1990). Visión de la investigación en Chile a través de algunos indicadores epistemométricos. Archivos de Biología y Medicina Experimentales, 64, 51-64.

Lillo, S. \& Martini, N. (2013). Principales Tendencias Iberoamericanas en Psicología Clínica. Un Estudio Basado en la Evidencia Científica. Terapia Psicológica, 31(3), 363-371. DOI: 10.4067/S0718-48082013000300011.
Moyano, E. (1999). Hacia la integración de métodos cuantitativos y cualitativos en la investigación social. Revista de la facultad de humanidades de Universidad de Santiago de Chile, 4, 1-20.

Moyano, E. \& Moyano, A. (1996) Panorama de la investigación psicológica publicada en Chile. Interdisciplinaria Revista de psicología y ciencias afines. Journal of psychology and related sciences,13(2), 79-95.

Moyano, E. \& Ramos, N. (2000). Contexto y Evaluación de la Investigación Psicológica en el Chile de Fin de Siglo. Psykhe, 9(1), 63-75.

Opazo, R. (1992) Integración en psicoterapia, Roberto Opazo ed., Cecidep, Santiago de Chile.

Polanco-Carrasco, R., Gallegos, M., Salas, G., \& López-López, W. (2017). Las revistas de psicología en Chile: historia y situación actual. Terapia Psicológica, 35, 81-93. DOI: 10.4067/ S0718-48082017000100008.

Rey, C., Martínez, J., \& Guerrero, S. (2009). Tendencias de los Artículos en Psicología Clínica en Iberoamérica. Terapia Psicológica, 27(5), 61-71. DOI: 10.4067/S0718-48082009000100006

Riffo, G. (2011). Tendencia de los artículos de Psicología Clínica en Chile, Latinoamérica y España. Salud \& Sociedad, 2(1), 90-99. DOI: 10.22199/S07187475.2011.0001.00006

Rodríguez, A. (2004). La investigación de resultados y el futuro de la psicoterapia: alternativas a los tratamientos empíricamente validados. Papeles del Psicólogo, 1-16.

Rull, J. \& Pereda, N. (2011). Revisión sistemática de los programas de tratamiento psicológico aplicados a víctimas adultas de abuso sexual en la infancia. Anuario de Psicología, 41(1-3), 81-105.

Salas, G., Ponce, F. P., Méndez-Bustos, P., VegaArce, M., Pérez, M., ... Cárcamo-Vásquez, H. (2017). 25 Años de Psykhe: Un Análisis Bibliométrico. Psykhe, 26(1), 1-17. DOI: 10.7764/ psykhe.26.1.1205 
Sales, C. (2009). Aspectos metodológicos de la investigación de la Psicoterapia. Panorama histórico. Revista de la Asociación Española de Neuropsiquiatría, XXIX, 383-403.

Sierra Bravo, R. (1987). Técnicas de investigación social. Teoría y Ejercicios. Ed. Amorrortu.

Vera-Villarroel, P. \& Lillo, S. (2006). La Investigación actual en Psicología Clínica en Chile: Un análisis a partir de la Producción. Terapia Psicológica, 24, 221-230.

Vera-Villarroel, P. \& Mustaca, A. (2006). Investigaciones en Psicología Clínica basadas en la evidencia en Chile y Argentina. Revista Latinoamericana de Psicología, 38(3), 551-565. 